\title{
CONSTRUCTING ECOLOGIES
}

\author{
Roger Cropp $^{1^{*}} \&$ John Norbury ${ }^{2}$
}

${ }^{1}$ Atmospheric Environment Research Centre, Griffith University, Nathan, Queensland, 4111, Australia

${ }^{2}$ Mathematical Institute, University of Oxford, 24-29 St. Giles, Oxford OX1 3LB, UK

* Corresponding author: email: $\quad$ r.cropp@griffith.edu.au

Phone: $\quad+61737354036$

Fax: $\quad+61737357459$

Post: $\quad$ Atmospheric Environment Research Centre

Griffith School of Environment

170 Kessels Road

Nathan, Queensland 4111

Australia 


\begin{abstract}
We synthesise the generic properties of ecologically realistic multi-trophic level models and define criteria for ecological realism. We define an "ecospace" in which all ecologically realistic dynamics are confined, and construct "resource rays" that define the resources available to each species at every point in the ecospace. Resource rays for a species are lines from a vertex of maximum resource to the opposite boundary where no resources are available. The growth functions of all biota normally decrease along their resource rays, and change sign from positive to negative. This property prescribes that each species must have a zero isosurface within the ecospace. We illustrate our conditions on a highly cited three trophic level model from population dynamics, showing how to extend this system biologically consistently to a closed ecological system. Our synthesis extends the concept of carrying capacity of population models to explicitly include exhaustion of limiting resources, and so allows for population biology models to be considered as ecologically closed systems with respect to a key limiting nutrient. This approach unifies many theoretical and applied models in a common biogeochemical framework, facilitates better understanding of the key structures of complex ecologies, and suggests strategies for efficient design of experiments.
\end{abstract}

\title{
Keywords
}

Conceptual framework resource space plankton consistent ecology 


\section{Introduction}

Complex marine plankton models that explicitly resolve the interactions between several functional types or trophic levels are being developed for applications in climate prediction (i.e. PlankTOM10 (Le Quéré et al., 2005); (Moore et al., 2002)) and fisheries management (i.e. NEMURO (Kishi et al., 2007)). At least one limiting nutrient and three trophic levels or plankton functional types are typically included in such models. They tend to approximately conserve the quantity of these nutrients, and hence run in an "approximately closed" manner. This is consistent with the view that export production in the ocean is generally balanced by new production (Eppley and Peterson, 1979; Sarmiento and Gruber, 2006), and that plankton ecosystems therefore approximately conserve mass. Similar views are expressed in terrestrial ecology, with Loreau (2010) noting "Without recycling of materials in limited supply, energy flow would stop rapidly, leading to ecosystem collapse....", and Schmitz (2010) confirming that ecosystems in general are largely selfcontained and that material is largely recycled within a system. Similarly, many ecological experiments, from small-scale measurements of grazing to large-scale mesocosm experiments are done in "exactly closed" environments.

We explain our approach by considering idealised ecosystem models with three trophic-levels that are exactly closed with respect to a limiting nutrient, in which the populations are measured as concentrations of this nutrient. The assumption of exact conservation of mass permits our theory to be developed, but does not restrict its practical importance as this assumption may later be relaxed without loss of understanding. We compare two conceptual approaches with these models: a 'population dynamics' approach that constrains the lowest trophic level with a carrying capacity, and an 'ecosystem' approach that conserves mass. An objective of our approach is to bridge these two areas that have 
developed essentially independently (see discussions in Loreau, 2010; May and McLean, 2007; Pastor, 2008).

There are many studies of predator/prey systems in population biology/ecology, however, few if any of these studies include the effect of exhausting the limiting nutrient and, in fact, most use a version of the carrying capacity concept to constrain the growth of the lowest trophic level. Loreau (2010) argues that carrying capacity is analogous to nutrient conservation, but in the form commonly implemented in population models (for example Hastings and Powell (1991), McCann and Yodzis (1995), Deng (2004)) the higher trophic levels do not influence the availability of nutrient, yet they contain nutrient that is then unavailable to the lowest trophic level.

We present a simple way to include the effect of a limiting nutrient and create closed ecosystem models that are both ecologically realistic and internally consistent. This approach allows most population biology models to be incorporated into an ecosystem framework. We formulate a simple 'limiting resource' criterion for each trophic level to prey on the one below it; this allows a straightforward and ecologically consistent procedure for adding trophic levels to models. This limiting resource approach reveals that the lack of a limiting nutrient is a fundamental inconsistency in most predator/prey population models.

We propose a "reflective lid" condition that must be placed on the limiting nutrient. This reflective lid condition may be expressed simply as $\dot{N}>0$ on $N=0$ (where $N$ is the amount of inorganic or "free" nutrient in the system) analogously to the implicit resource limitation constraint of all the other trophic levels. We describe the lid as "reflective" to stress that when the inorganic nutrient is almost exhausted the system behaviour is severely restricted by the presence of the zero nutrient value lid.

We observe that most existing predator/prey systems, including most plankton models, already have a version of this reflective lid behaviour when the predator's resource 
(the prey) nears exhaustion. We extend this (usually implicitly modelled) behaviour of the predator to the prey by observing that an autotroph taking up an inorganic nutrient is the only internally-consistent lowest trophic level for any model, and the ecosystem is viably closed as it is the fate of all the higher trophic levels to ultimately (after death) be recycle their organic nutrient back into inorganic nutrient.

We consider idealized, isolated models that exactly conserve mass, as a constant total mass $\left(N_{T}\right)$ simply defines the ecospace and hence the resource spaces. Once a consistent model is obtained, the exact conservation of mass constraint may be relaxed without compromising the model's consistency or our understanding of its properties. In this case, rather than $N_{T}$ being a constant it may vary according to the known flux of nutrient across the boundaries of the model. This results in the mass-conservation lid moving over time and hence continually modifying the ecospace. However, the important point is that an ecospace is always defined, and this approach is therefore useful for modelling real systems that do not exactly conserve nutrients over all time and space scales.

Our aim is to produce simple conditions ("ecological axioms") that allow trophic levels to be consistently added in a biologically reasonable manner. This eases the construction, and checking, of the consistency of large computer models of self-contained ecologies. We use our axioms to examine simple Lotka-Volterra systems that use a carrying capacity and demonstrate an inconsistency that fundamentally separates one- and two-trophic level models of this type from those with three or more trophic levels. We then show that the many interesting results of population dynamics may be observed in these more realistic ecological models, but may be modified significantly by exhaustion of the limiting resource. 


\section{Conditions}

\subsection{Ecospace}

We first rigorously define an ecological space (ecospace, $E$, Fig 1(a)) in which all the ecologically relevant dynamics of the system, in this case the "life histories" of the concentrations of the various plankton, can occur. Our first tenet is that the net growth rates of population variables $x_{i}(t)$, where $t$ is time, should be measurement invariant, that is they should not depend on the way in which the population is measured, so $\frac{1}{x_{i}} \frac{d x_{i}}{d t}$ is some continuous function of the variables it depends on over closed intervals $0 \leq x_{i} \leq N_{T}$ where $N_{T}$ is the total amount of nutrient in the system. As usual we measure the populations of the plankton functional types by $x_{i}$, the amount of key, or limiting, nutrient that is in type $i$. Thus, the per capita growth rates, for $x_{i}>0$, are given by

$$
\frac{1}{x_{i}} \frac{d x_{i}}{d t}=f_{i}\left(x_{1}, x_{2}, \cdots, x_{n} ; N\right)
$$

where $f_{i}$ is continuous in its arguments, which include the amount of available inorganic nutrient $(N)$. We describe the $x_{i}$ as "active" variables because they grow or die as a proportion of their population size, and hence "control" their own dynamics. The critical implication of this rule is that each $f_{i}$ should be well defined as $x_{i}$ tends to the zero population level.

We only consider smooth processes where each $\frac{\partial f_{i}}{\partial x_{j}}$ exists continuously in $0 \leq x_{i} \leq N_{T}$. The closed intervals $0 \leq x_{i} \leq N_{T}$ form an ecological domain that contains all the ecologically realistic behaviour of the system including extinction, which occurs when $x_{i}=0$. This domain also includes theoretically important but ecologically unrealistic states 
such as unachievable eco-world dominance, when $x_{i}=N_{T}$ and all other populations are extinct. The $x_{i}$ are measured in a currency of the limiting or primary nutrient, and the total mass of this nutrient is conserved, which means that the total amount of organic nutrient in the marine population is always equal to that consumed from the total $N_{T}$ in the ecology, that is:

$$
x_{1}+x_{2}+\cdots+x_{n}=N_{T}-N \text {. }
$$

This implies an equivalent condition on the $f_{i}$. We differentiate (2) and use (1), which gives:

$$
x_{1} f_{1}+x_{2} f_{2}+\cdots+x_{n} f_{n}=-\frac{d N}{d t} .
$$

In practice for plankton systems, conservation arises because the net rate of supply/consumption of the inorganic nutrient $\frac{d N}{d t}$ is in the form of (3), which by integration using the Kolmogorov equations (1) gives us (2). We note that $N$ is a "passive" variable because its own population size does not always influence the rate at which $N$ changes.

It is often convenient to discuss the populations in terms of the fraction of the total amount of nutrient that each population represents. Each population is measured by $\hat{x}_{i}=\frac{x_{i}}{N_{T}}$, with $\hat{N}=\frac{N}{N_{T}}$, and then (2) becomes:

$$
\hat{x}_{1}+\hat{x}_{2}+\cdots+\hat{x}_{n}=1-\hat{N} \text {. }
$$

We now use equation (4) to eliminate $N$ from equation (1): this turns the population biology model given by equation (1) for a fixed $N$ into an ecology with a finite value of limiting resource. From now on we drop the hats and define the ecospace

$$
E \equiv\left\{0<x_{1}+x_{2}+\ldots+x_{n}<1 ; 0<x_{i} \forall i\right\}
$$

Hence the variables are effectively fractions of the key limiting nutrient, which is typically nitrogen for marine plankton systems. 
The conservation of mass condition forms an "ecological lid" at $N=0$; when $\frac{d N}{d t}>0$ on $N=0$ we call this the "reflective lid" condition, and the dynamics of the system are constrained to lie below the lid. The lid is shown in Fig 1(a) as the triangular face in the plane of the page labelled $N=0$. Note that $\frac{d N}{d t}>0$ on the ecological lid stipulates that some biota must die and re-mineralise to $N$ when the system is in this state; thus the autotroph can grow and the system is kept functioning. As $N$ is passive it can (and must) grow when $N=0$, but if any active variable goes extinct at time $t_{*}$, say $x_{i}\left(t_{*}\right)=0$ for some $t_{*}$ then $x_{i}(t) \equiv 0$ for all time. Hence life histories of plankton systems are orbits or trajectories $\left\{x_{1}(t), x_{2}(t), \cdots, x_{n}(t)\right\}$ that move in the ecospace $E$ as time evolves; orbits only intersect at equilibrium (equivalently fixed or critical) points where $\frac{d x_{i}}{d t}=0$ for all $i$. Population dynamics usually focuses only on the living variables $x_{i}$; it is the ecology of a closed system that requires us to also monitor the inorganic nutrient $N$, which can play a vital role in the system dynamics, especially when $N$ becomes small.

\subsection{Resource Space}

All living organisms require resources for their continued existence. The resources for heterotrophic species in population and ecosystem models are the species upon which they feed. The limiting resources for these species are therefore explicitly represented in the models, and we may define resource spaces for these species analogously to our definition of an ecospace $E$. The resource spaces for species that consume other species represented explicitly in the model occupy the same space as the ecospace but differ in that they view the ecospace $E$ from the perspective of that heterotroph, effectively the origins of the resource 
spaces are located at different vertices of the ecospace. An $n$ trophic level model will have $n$ explicit resource spaces defined by the model, which we label $E^{1}$ through to $E^{n}$ (Fig 1).

The resource space $E^{i}$ of species $x_{i}$ has its origin at the point in the ecospace where that resource is maximum, that is, if $x_{i}$ feeds upon $x_{j}$, then its resource space is defined by:

$$
E^{i}=\left\{r_{1}^{i}, r_{2}^{i}, \cdots, r_{n}^{i}\right\}=\left\{x_{1}, x_{2}, \cdots, x_{j-1}, 1-x_{j}, x_{j+1}, \cdots, x_{n-1}, x_{n}\right\} \text { for } 1 \leq i, j \leq n
$$

The $1-x_{j}$ term in equation (6) reflects that this axis of the resource space points in the opposite direction to the corresponding axis of the ecospace as the origin of the resource space is located at the point of maximum resource, where $x_{j}=1$. For a consistent ecology, each species must have a resource explicitly defined within the system. The species occupying the lowest trophic level in the model, say $x_{1}$, must be an autotroph that utilises inorganic nutrient $N$ as a resource. The resource space for such an autotroph is not obviously defined by this scheme, as inorganic nutrient is not explicitly represented in the system due to conservation of mass. However, the resource space for the autotroph $x_{1}\left(E^{1}\right)$ is just the ecospace $E$, as from the definition of the ecospace inorganic nutrient $N$ is a maximum at the origin and is zero at the lid. These conditions do not necessarily preclude $x_{1}$ from being a mixotroph and also consuming other organisms, which are represented by a separate resource space.

\subsubsection{Resource Rays}

We now define resource rays that span a resource space $\left(E^{i}\right)$. The rays emanate from the origin of $E^{i}$ (which is the relevant pure resource vertex of $E$ where the resource $R^{i}=x_{i-1}$ is maximal) to the opposite face where the relevant resource level $R^{i}=x_{i-1}=0($ Fig $1(\mathrm{~b}, \mathrm{c}))$. The biological interpretation of the rays is that available resources (inorganic nutrient in the 
case of autotrophs, but lower trophic level organisms in the case of grazers and carnivores) reduce as one moves from the origin of the ray to the other side. We note that the resource rays for the lowest trophic level explicitly represented in any model originate from the origin of the state space, but will only have a zero resource face (as the higher trophic levels do) if the system conserves mass of nutrient. This fundamental difference can lead to subtle inconsistencies in ecological models when conservation of nutrient is not explicitly modelled.

\subsubsection{Resource Ray Gradient Condition}

At each point on a resource ray (say for resource $R^{i}$ ), we describe the relevant $x_{i}$ population's ability to thrive on the quantity of its resource available there by its life function $f_{i}$, see (1). Here each resource ray spanning the resource space $E^{i}$ is written ${\underset{\sim}{r}}^{i}=\left(r_{1}^{i}, \cdots, r_{n}^{i}\right) \in E^{i}$ where ${\underset{\sim}{r}}^{i}=\left(x_{1}, \cdots, x_{j-1}, 1-x_{j}, x_{j+1}, \cdots, x_{n}\right)$, where we assume that $x_{i}$ feeds on $x_{j}$. Each life function $f_{i}$, for conventional biology, decreases along its resource rays and the directional derivative of a life function along its resource rays must therefore be negative. This may be stated formally as:

$$
\begin{aligned}
\stackrel{r}{i}^{i} \cdot \nabla f_{i} & \equiv r_{1}^{i} \frac{\partial f_{i}}{\partial r_{1}^{i}}+r_{2}^{i} \frac{\partial f_{i}}{\partial r_{2}^{i}}+\ldots+r_{n}^{i} \frac{\partial f_{i}}{\partial r_{n}^{i}}<0 \\
& \equiv x_{1} \frac{\partial f_{i}}{\partial x_{1}}+\ldots+x_{j-1} \frac{\partial f_{i}}{\partial x_{j-1}}+\left(1-x_{j}\right) \frac{\partial f_{i}}{\partial\left(1-x_{j}\right)}+x_{j+1} \frac{\partial f_{i}}{\partial x_{j+1}}+\ldots+x_{n} \frac{\partial f_{i}}{\partial x_{n}}<0,(7) \\
& =x_{1} \frac{\partial f_{i}}{\partial x_{1}}+\ldots+x_{j-1} \frac{\partial f_{i}}{\partial x_{j-1}}-\left(1-x_{j}\right) \frac{\partial f_{i}}{\partial x_{j}}+x_{j+1} \frac{\partial f_{i}}{\partial x_{j+1}}+\ldots+x_{n} \frac{\partial f_{i}}{\partial x_{n}}<0
\end{aligned}
$$

where the $r_{j}^{i}$ are the axes of the resource space $E^{i}$ as shown in the example in Fig 1(c). This relation (7) is the simplest analytical way to check for normal life function behaviour.

Remark: we observe that organisms that cooperate have the potential to create exceptions to these ray conditions. For instance, we might consider an intra-species cooperative process that overcomes the effect of declining resources on the growth rate. Such exceptions may occur, 
for example, in a species that cooperatively hunts, where there is an optimum size for hunting efficiency. These more interesting ecologies will not be considered here as they would require the resource ray gradient condition to be relaxed in specific regions of the resource space.

\subsubsection{The Resource Ray Sign Condition}

A realistic ecosystem model must have the value of each life function $f_{i}$ positive at its maximum resource vertex, that is the origin $\left(R^{i}=1\right)$ of its resource space $E^{i}$, and negative at its zero resource face ( $\left.R^{i}=0\right)$, that is the face opposite to that origin (Fig 1). Therefore, additionally to $f_{i}$ decreasing along any ray from its origin to its zero-resource face or lid (equation (7)), each life function $f_{i}$ must change sign along every such ray. This may be stated formally as:

$$
f_{i}\left(R_{i}=1\right)>0>f_{i}\left(R_{i}=0\right) .
$$

The positive part of this condition $f_{i}\left(R_{i}=1\right)>0$ means that a species' growth rate on maximum resources must exceed its intrinsic death rate, and hence this condition constrains the magnitude of the natural mortality rate of each species. If this condition is not met the species does not survive in that environment, and there is little point in including it in the model. Conversely, the condition that $0>f_{i}\left(R_{i}=0\right)$ means that in the absence of resources the species must die.

The key ecological point of this condition is that each life function $f_{i}$ must be negative everywhere on its zero resource face, otherwise the model claims that the species can grow even though it has no resources to fuel its growth. Population models are constructed on the premise that higher trophic levels feed on lower trophic levels, and the higher trophic level populations in such models typically satisfy this condition. However, we shall show that this condition is often not met for the first trophic level in population models with three or more 
trophic levels that use a carrying capacity to provide "ecological realism". This reveals a subtle inconsistency in the theoretical basis of such models.

\subsection{Zero Isosurfaces}

Along every resource ray $\underset{\sim}{\underset{\sim}{i}}$ in its resource space $E^{i}$ there exists a point $r_{*}^{i}=\lambda_{*}^{i}\left(r_{1}^{i}, \cdots, r_{n}^{i}\right)$, with $0<\lambda_{*}^{i}<1$, where the growth and loss terms of the population $x_{i}$ balance (i.e. $f_{i}\left({\underset{\sim}{*}}_{*}^{i}\right)=0=\frac{d x_{i}}{d t}$; note $\lambda_{*}^{i}$ varies as we vary the ray). These points ${\underset{\sim}{*}}_{*}^{i}$ then form a surface in $E$ that we call the $f_{i}$ zero isosurface (i.e. $f_{i}=0$ in $E$, the planes in Fig 1(c)). The key attribute of an isosurface is that it must divide the ecospace $E$ into two parts, one part where $x_{i}(t)$ is increasing in $t$, and one where $x_{i}(t)$ is decreasing in $t$. Each such isosurface ( $\left.f_{i}=0\right)$ that complies with the ray conditions creates a closed part of $E$ around the point of maximum resource $\left(x_{1}=0, \cdots, x_{j}=1, \cdots, x_{n}=0\right)$ for that species $x_{i}$; in this closed part $x_{i}(t)$ is increasing. These isosurfaces may or may not intersect in the ecological domain. While it is possible to derive conditions for specific models that determine whether none, one, several or all of the zero isosurfaces intersect, to be brief we shall only consider the simplest realistic ecologies in which all the zero isosurfaces intersect.

\section{Lotka-Volterra Examples}

Lotka-Volterra models represent the simplest case of ecosystem models as they have planar isosurfaces. Although these models are now considered too simplistic for simulation models, they have been a mainstay of theoretical ecology and population biology (Loreau, 2010; May and McLean, 2007; Pastor, 2008). Many studies of population dynamics use logistic Lotka-Volterra (LLV) models, where the models have been cast in a more ecologically realistic form by constraining the lowest trophic level to logistic growth by using 
a carrying capacity. In the following examples we shall define for comparison mass conserving Lotka-Volterra models (which we shall call CLV models) by taking the growth rate $(r)$ of the autotroph to depend linearly on the amount of available inorganic nutrient (i.e. $r=r N)$.

\subsection{One Trophic Level}

We consider a mass conserving Lotka-Volterra (CLV) model with one trophic level:

$$
\begin{aligned}
& \dot{x}_{1}=\hat{r} N x_{1}-d_{1} x_{1} \\
& \dot{N}=d_{1} x_{1}-\hat{r} N x_{1}
\end{aligned} .
$$

Conservation of mass means $\frac{d x_{1}}{d t}+\frac{d N}{d t}=0$ or equivalently $x_{1}+N=N_{T}$. We may rewrite equation (9) as:

$$
\dot{x}_{1}=\hat{r}\left(N_{T}-x_{1}\right) x_{1}-d_{1} x_{1} .
$$

This model satisfies our ray conditions and the positive sign condition places an upper bound on the mortality rate $d_{1}<\hat{r} N_{T}$ (Fig 2(a)).

It is well known that the one trophic level mass conserving Lotka-Volterra (CLV) model is equivalent to the one trophic level LLV model (Loreau, 2010). Equation (10) is equivalent to:

$$
\dot{x}_{1}=r x_{1}\left(1-\frac{x_{1}}{K}\right)
$$

where $K=N_{T}-\frac{d_{1}}{\hat{r}}$ and $r=\hat{r} K$. Both the CLV and LLV one trophic level models comply with our conditions for ecological realism.

\subsection{Two Trophic Levels}

The two trophic level CLV model is written: 


$$
\begin{aligned}
& \dot{x}_{1}=\hat{r} N x_{1}-\hat{c}_{1} x_{1} x_{2}-d_{1} x_{1} \\
& \dot{x}_{2}=\hat{c}_{1} x_{1} x_{2}-d_{2} x_{2} \\
& \dot{N}=d_{1} x_{1}+d_{2} x_{2}-\hat{r} N x_{1}
\end{aligned}
$$

Using $\frac{d x_{1}}{d t}+\frac{d x_{2}}{d t}+\frac{d N}{d t}=0 \Leftrightarrow x_{1}+x_{2}+N=N_{T}$, we rewrite (12) as:

$$
\begin{aligned}
& \dot{x}_{1}=\hat{r}\left(N_{T}-x_{1}-x_{2}\right) x_{1}-\hat{c}_{1} x_{1} x_{2}-d_{1} x_{1} \\
& \dot{x}_{2}=\hat{c}_{1} x_{1} x_{2}-d_{2} x_{2}
\end{aligned}
$$

Equation (13) meets our criteria for ecological realism as it complies with our ray conditions and analogously to the one trophic level model the positive ray conditions place upper bounds on the mortality rates: $d_{1}<\hat{r} N_{T}$ and $d_{2}<c_{1} N_{T}$ (Fig 2(b, c) respectively). The two trophic level CLV model may be rewritten as the LLV system:

$$
\begin{aligned}
& \dot{x}_{1}=r x_{1}\left(1-\frac{x_{1}}{K}\right)-c_{1} x_{1} x_{2}, \\
& \dot{x}_{2}=c_{1} x_{1} x_{2}-d_{2} x_{2}
\end{aligned}
$$

where $K=N_{T}-\frac{d_{1}}{\hat{r}}, r=\hat{r} K$ and $c_{1}=\hat{r}+\hat{c}_{1}$. Both the CLV and LLV two trophic level models are therefore ecologically consistent and realistic, and we observe that $K$ is the carrying capacity of $x_{1}$ in the absence of $x_{2}$.

\subsection{Three Trophic Levels}

The three trophic level CLV model is:

$$
\begin{aligned}
& \dot{x}_{1}=\hat{r} N x_{1}-\hat{c}_{1} x_{1} x_{2}-d_{1} x_{1} \\
& \dot{x}_{2}=\hat{c}_{1} x_{1} x_{2}-\hat{c}_{2} x_{2} x_{3}-d_{2} x_{2}, \\
& \dot{x}_{3}=\hat{c}_{2} x_{2} x_{3}-d_{3} x_{3} \\
& \dot{N}=d_{1} x_{1}+d_{2} x_{2}+d_{3} x_{3}-\hat{r} N x_{1}
\end{aligned}
$$

which may be rewritten using $\frac{d x_{1}}{d t}+\frac{d x_{2}}{d t}+\frac{d x_{3}}{d t}+\frac{d N}{d t}=0 \Leftrightarrow x_{1}+x_{2}+x_{3}+N=N_{T}$ as 


$$
\begin{aligned}
& \dot{x}_{1}=\hat{r}\left(N_{T}-x_{1}-x_{2}-x_{3}\right) x_{1}-\hat{c}_{1} x_{1} x_{2}-d_{1} x_{1} \\
& \dot{x}_{2}=\hat{c}_{1} x_{1} x_{2}-\hat{c}_{2} x_{2} x_{3}-d_{2} x_{2} \\
& \dot{x}_{3}=\hat{c}_{2} x_{2} x_{3}-d_{3} x_{3}
\end{aligned}
$$

The ray gradient conditions for all the $f_{i}$ are satisfied and the positive ray conditions again place upper bounds on the magnitude of the mortality rates: $d_{1}<\hat{r} N_{T}, d_{2}<\hat{c}_{1} N_{T}$, and $d_{3}<\hat{c}_{2} N_{T}$ (Fig 3(a)). All species therefore satisfy our ray conditions everywhere in the state space provided the mortality terms for each are sensible, that is, allow them to grow on maximal resources.

The three trophic level CLV model may be written in LLV form as:

$$
\begin{aligned}
& \dot{x}_{1}=r x_{1}\left(1-\frac{x_{1}}{K}\right)-c_{1} x_{1} x_{2}-\frac{r}{K} x_{1} x_{3} \\
& \dot{x}_{2}=c_{1} x_{1} x_{2}-c_{2} x_{2} x_{3}-d_{2} x_{2} \\
& \dot{x}_{3}=c_{2} x_{2} x_{3}-d_{3} x_{3}
\end{aligned}
$$

where $K=N_{T}-\frac{d_{1}}{\hat{r}}, r=\hat{r} K$. and $c_{1}=\hat{r}+\hat{c}_{1}$. This model is not equivalent to the usual three trophic level LLV model (Deng, 2004; Hastings and Powell, 1991; Rosenzweig, 1973):

$$
\begin{aligned}
& \dot{x}_{1}=r x_{1}\left(1-\frac{x_{1}}{K}\right)-c_{1} x_{1} x_{2} \\
& \dot{x}_{2}=c_{1} x_{1} x_{2}-c_{2} x_{2} x_{3}-d_{2} x_{2}, \\
& \dot{x}_{3}=c_{2} x_{2} x_{3}-d_{3} x_{3}
\end{aligned}
$$

as the LLV model does not include the interaction term $\hat{r} x_{1} x_{3}=(r / K) x_{1} x_{3}$. The LLV model is only consistent with the CLV model if $x_{1}$ and $x_{3}$ have an amensal relationship, that is, the presence of $x_{3}$ is detrimental to $x_{1}$ but $x_{3}$ gains no benefit from the interaction. In contrast, the inclusion of such an interaction term in a mass conservation context represents that the nutrient assimilated into the $x_{3}$ population is not available to fuel $x_{1}$ 's growth. The implication of the absence of this term from LLV models becomes apparent by applying our ray conditions. 
The ray conditions for $f_{2}$ and $f_{3}$ of the LLV model (Fig 3(b) second and third boxes) are the same as for the CLV model (Fig 3(a)), and the resource rays for $f_{1}$ originate from the origin of $E$, as previously. The ray gradient condition $\underset{\sim}{x} \cdot \nabla f_{1}=-\frac{r}{K} x_{1}-c_{1} x_{2}<0$ is satisfied, and the positive ray sign condition is satisfied at the origin $f_{1}(0)=r>0$. The negative ray sign condition for $f_{1}$ is $0>r\left(1-\frac{x_{1}}{K}\right)-c_{1} x_{2}$, which is only satisfied where $\frac{r}{K} x_{1}+c_{1} x_{2}>r$ (Fig 3(b) first box). This condition is independent of $x_{3}$, therefore the isosurface $f_{1}=0$ does not form a closed subspace around the point of maximum resource, and the model is internally inconsistent. This inconsistency somewhat counter-intuitively results in the population of $x_{3}$ being unbounded, as is evident in the life histories in Fig 3(b). The dynamics of the Lotka-Volterra system changes substantially as a result of resolving this inconsistency (cf Fig 3 (a) with (b)), however, this is not necessarily the case, as we shall demonstrate using the 'teacup' model of Hastings and Powell (1991).

\section{The Hastings and Powell (1991) example}

We take a popular three trophic level food web model of Hastings and Powell (1991) that has been subject to considerable interest over the past twenty years (McCann and Yodzis, 1995; Rinaldi and Muratori, 1992; Yu et al., 1996). The bifurcation structure of this model has recently been analysed in detail by Deng and Hines (Deng, 2001; Deng, 2004; Deng and Hines, 2002; Deng and Hines, 2003) with a particular aim of showing different chaotic behaviours for certain parameter regimes. The model, after scaling time by the intrinsic growth rate of $x$, and $x, y$ and $z$ by the carrying capacity of $x$, is:

$$
\frac{d x}{d t}=x(1-x)-\frac{a_{1} x y}{1+b_{1} x}
$$




$$
\begin{gathered}
\frac{d y}{d t}=y\left(\frac{a_{1} x}{1+b_{1} x}-\frac{a_{2} z}{1+b_{2} y}-d_{1}\right), \\
\frac{d z}{d t}=z\left(\frac{a_{2} y}{1+b_{2} y}-d_{2}\right)
\end{gathered}
$$

We utilise parameter values nominated by Hastings and Powell (1991), given in Table 2, in our numerical simulations, which give the famous 'teacup attractor' they described (Fig 4(a)). The ray gradient conditions are satisfied for all $f_{i}$, and the ray sign conditions are satisfied for $f_{2}$ and $f_{3}$. The positive ray sign condition is met for $f_{1}$, but the negative ray sign condition is not, as $f_{1}\left(R^{1}=0\right)=1-x-\frac{a_{1} y}{1+b_{1} x}<0$ is only satisfied for $y>\frac{(1-x)\left(1+b_{1} x\right)}{a_{1}}$ (Fig 4(a), first box, third line). As for the three trophic level LLV model above, this condition does not satisfy our requirement that each isosurface forms a closed space about the point of maximum resource. Although $f_{1}$ contains an explicit term designed to confer ecological realism through the inclusion of a carrying capacity, $f_{1}$ is inconsistent with the other equations in the model as it effectively frees $z$ from its apparent resource limitation.

The model orbits in Fig. 4(a) can penetrate the scaled resource level of the ecosystem because there is no lid condition in the model, with the $f_{1}$ isosurface clearly showing that $x$ can grow regardless of how much nutrient is sequestered in the $z$ population. This again results in the population of the highest trophic level $(z)$ being effectively unbounded.

The Hastings and Powell model may be put into a consistent ecosystem form if we introduce a limiting inorganic nutrient $(N)$ and half-saturation constant $(\varepsilon)$ as follows:

$$
\begin{aligned}
& \frac{d x}{d t}=x\left(\frac{N}{N+\varepsilon}-x\right)-\frac{a_{1} x y}{1+b_{1} x}, \\
& \frac{d y}{d t}=y\left(\frac{a_{1} x}{1+b_{1} x}-\frac{a_{2} z}{1+b_{2} y}-d_{1}\right),
\end{aligned}
$$




$$
\frac{d z}{d t}=z\left(\frac{a_{2} y}{1+b_{2} y}-d_{2}\right) .
$$

Here we choose a simple Michalis-Menten (Holling type II) modification of the growth rate coefficient so that at small values of the inorganic nutrient $(N<\varepsilon)$ the growth rate linearly depends on the amount of inorganic nutrient available. The ray gradient conditions are met for all $f_{i}$ as before, as are the positive ray sign conditions and the negative ray conditions for $f_{2}$ and $f_{3}$ (Figure $3(\mathrm{~b})$ ). The negative ray sign condition for $f_{1}$ is now $f_{1}\left(R^{1}=N=0\right)=-x-\frac{a_{1} y}{1+b_{1} x}$ which is negative everywhere in the ecospace.

This "closed population dynamics" model now satisfies the ecology conditions (above axioms) with the only difference to the CLV model being that the planar isosurfaces are curved. Figure 4(b) shows that orbits cannot penetrate the scaled resource level of the ecosystem due to the cap of the limiting nutrient being imposed. The isosurface folds over so that $f_{1}$ is strictly negative on the reflective lid; the influence of the reflective lid on the dynamics is then to bend the orbits, or life histories, of the populations (the 'handle' of the teacup attractor) to keep them under the lid.

We note that we have used a small value of $\varepsilon$ (Table 2) for the simulation in Fig 4(b) to demonstrate that a smooth transition between inconsistent and consistent formulations is possible, and that population models do not necessarily lose their interesting properties. However, as $\varepsilon$ is increased the chaotic attractor is replaced by a stable equilibrium point that moves to a distribution of mass more consistent with the concept of a trophic pyramid.

\section{Conclusions}

We define criteria for the construction of consistent ecologies in which the life functions that describe the growth, predation and mortality of each species, guild or functional 
type are constrained by generic principles. These three basic principles of independence of scale, conservation of mass and explicit resource limitation may be considered ecological axioms that ensure realistic ecologies. In the real world no ecosystem exactly conserves mass (although laboratory and mesocosm experiments may impose this), however, imposition of exact mass conservation may provide deeper insight in a similar manner that assumptions of exact linearity have provided for many systems.

We implement explicit resource limitation by defining resource rays, and propose conditions along rays from each of the $x_{i}$ trophic level's maximum resource vertex (generally $\left.x_{i-1}=N_{T}=1\right)$ of the ecospace $E$. This condition says that the trophic function $f_{i}$ must decrease from positive to negative values as we move along each such ray in ecospace. Checking the ray conditions that $f_{i}$ decreases from positive to negative values along each ray allows complex, multi-trophic level models to be readily checked for ecological consistency. Along every ray there exists a point where the growth and loss terms of the population's $f_{i}$ balance (i.e. $f_{i}\left(R_{*}^{i}\right)=0=\frac{d x_{i}}{d t}$ ); these zero net resource points form an isosurface in $E$ that creates a closed subspace about its maximum resource vertex. When measured along any ray each isosurface lies at a distance $R_{*}^{i}$ from the face where the relevant resource equals zero, and $R_{*}^{i}$ is the minimum resource level required for the population $x_{i}$ to continuously exist. This is consistent with the $R^{*}$ theory that robustly predicts the winner of chemostat competition among phytoplankton (Tilman, 2007).

When we take the simplest biological life functions $f_{i}$ to be planar (i.e. Fig 3), we usually find just one stable equilibrium point in ecospace, about which the populations may oscillate. When we consider more interesting biological life functions, where the $f_{i}$ zero isosurfaces have become more curved (i.e. Fig 4), we may obtain life histories that become very complicated, as in the tea-cup attractor of the Hastings and Powell (1991) system. 
However, the presence of the reflective lid, at which the limiting resource becomes exhausted, may significantly modify the isosurfaces; this is shown for models with simple and complex life histories in Figures 3 and 4 respectively. Thus key experiments are required to determine the nature of the curvature of the zero isosurfaces. Such consistent ecological models then allow reliable computer modelling of ecological systems where the lowest trophic levels may be under increasing pressure from a changing environment, and humans are maximally exploiting the top trophic levels.

We view this approach as an adjunct to, rather than a replacement for, the usual fixed point approach. Analysis of the fixed points will not necessarily identify inconsistencies in a model that will be identified by this approach. The Lotka-Volterra and Hastings and Powell models are used only to demonstrate that the method easily and reliably identifies inconsistencies. We considered the Hastings and Powell model to make the point that imposing conservation of mass and explicit resource limitation does not necessarily mean that the interesting properties of a model are lost or even that the dynamics are significantly changed.

Our approach provides a unifying framework for understanding theoretical models that spans the divide between population dynamics and ecosystems. The models we consider here are simple and well-understood systems but it is in the construction and validation of complex ecosystem biogeochemical models being developed for applications in climate modelling and fisheries management that the advantages of these ecological axioms will be most evident. 


\section{Acknowledgements}

We wish to thank three anonymous reviewers for their thoughtful and constructive reviews of this work that have assisted us to clarify our thoughts and communications on this topic, and in this manuscript in particular.

\section{References}

Deng, B., 2001. Food chain chaos due to a junction-fold point. Chaos 11, 514-525.

Deng, B., 2004. Food chain chaos with canard explosion. Chaos 14, 1083-1092.

Deng, B., and Hines, G., 2002. Food chain chaos due to Shilnikov's orbit. Chaos 12, 533-538.

Deng, B., and Hines, G., 2003. Food chain chaos due to transcritical point. Chaos 13, 578585.

Eppley, R.W., and Peterson, B.J., 1979. Particulate Organic Matter Flux And Planktonic New Production In The Deep Ocean. Nature 282, 677-680.

Hastings, A., and Powell, T., 1991. Chaos In A Three Species Food Chain. Ecology 72, 896903.

Kishi, M.J., Kashiwai, M., Ware, D.M., Megrey, B.A., Eslinger, D.L., Werner, F.E., NoguchiAita, M., Azumaya, T., Fujii, M., Hashimoto, S., Huang, D., Iizumi, H., Ishida, Y., Kango, S., Kantakov, G.A., Kim, H.-c., Komatsu, K., Navrotsky, V.V., Smith, S.L., Tadokoro, K., Tsuda, A., Yamamura, O., Yamanaka, Y., Yokouchi, K., Yoshie, N., Zhang, J., Zuenko, Y.I., and Zvalinsky, V.I., 2007. NEMURO-a lower trophic level model for the North Pacific marine ecosystem. Ecol Model 202, 12-25.

Le Quéré , C., Harrison, S.P., Prentice, I.C., Buitenhuis, E.T., Aumonts, O., Bopp, L., Claustre, H., Cotrim da Cunha, L., Geider, R.J., Giraud, X., Klaas, C., Kohfeld, K.E., Legrende, L., Manizza, M., Platt, T., Rivkin, R.B., Sathyendranath, S., Uitz, J., Watson, A., and Wolf-Gladrow, D., 2005. Ecosystem dynamics based on plankton 
functional types for global ocean biogeochemistry models. Global Change Biology 11, 2016-2040.

Loreau, M., 2010. From Populations to Ecosystems. Princeton University Press, Woodstock, Oxfordshire.

May, R.M., and McLean, A., 2007. Theoretical Ecology: Principles and Applications. Oxford University Press, Oxford.

McCann, K.S., and Yodzis, P., 1995. Bifurcation structure of a three-species food chain model. Theor Popul Biol 48, 93-125.

Moore, J.K., Doney, S.C., Kleypas, J.A., Glover, D.M., and Fung, I.Y., 2002. An intermediate complexity marine ecosystem model for the global domain. Deep-Sea Research II 49, 403-462.

Pastor, J., 2008. Mathematical Ecology of Populations and Ecosystems. Wiley-Blackwell, Chichester.

Rinaldi, S., and Muratori, S., 1992. Slow-fast limit cycles in predator-prey models. Ecol Model 61, 287-308.

Rosenzweig, M.L., 1973. Exploitation in three trophic level models. Am Nat 107, 275-294.

Sarmiento, J., and Gruber, N., 2006. Ocean Biogeochemical Dynamics. Princeton University Press, Princeton.

Schmitz, O.J., 2010. Resolving Ecosystem Complexity. Princeton University Press, Woodstock, Oxfordshire.

Tilman, D., Interspecific competition and multispecies coexistence., in: May, R. M. and McLean, A., Eds.), Theoretical Ecology, Oxford University Press, Oxford 2007, pp. 257.

Yu, A., Kuznetsov, Y., and Rinaldi, S., 1996. Remarks on food chain dynamics. Math Biosci $133,1-33$. 
Tables

Table 1. Nondimensional parameters and parameter values used in numerical solutions of the Lotka-Volterra models for $N_{T}=1$.

Non-dimensional parameters LV model value

\begin{tabular}{lc}
\hline$r$ & 1 \\
$c_{1}$ & 0.3 \\
$c_{2}$ & 0.3 \\
$d_{1}$ & 0.1 \\
$d_{2}$ & 0.01 \\
\hline
\end{tabular}

Table 2. Non-dimensional parameters, scalings and parameter values used in numerical solutions of the Hastings and Powell (1991) model.

\begin{tabular}{ccc}
\hline Non-dimensional parameters & Scaling $^{*}$ & Parameter values \\
\hline$a_{1}$ & $\left(K_{0} A_{1}\right) /\left(R_{0} B_{1}\right)$ & 5.0 \\
$b_{1}$ & $K_{0} B_{1}$ & 3.0 \\
$a_{2}$ & $\left(K_{0} A_{2} C_{2}\right) /\left(C_{1} R_{0} B_{2}\right)$ & 0.1 \\
$b_{2}$ & $K_{0} /\left(C_{1} B_{2}\right)$ & 2.0 \\
$d_{1}$ & $D_{1} / R_{0}$ & 0.4 \\
$d_{2}$ & $D_{2} / R_{0}$ & 0.01 \\
$\varepsilon$ & & 0.000125
\end{tabular}

$R_{0}$ is the intrinsic growth rate of $x ; K_{0}$ is the carrying capacity of $x ; C_{1}$ and $C_{2}$ are the conversion rates of $x$ to $y$ and of $y$ to $z ; D_{1}$ and $D_{2}$ are the death rates of $y$ and $z ; A_{1}$ and $A_{2}$ are the grazing rates of $y$ on $x$ and $z$ on $y$; and $B_{1}$ and $B_{2}$ are the half-saturation constants for $y$ grazing on $x$ and $z$ grazing on $y . \varepsilon$ is introduced with the imposition of mass conservation. 


\section{Figure Legends}

Figure 1. (a) A three-species ecospace showing the lid at $N=0$ imposed by conservation of mass (dotted lines). (b) A typical resource space showing the maximum resource vertex at $R^{i}=1$ and the zero resource face opposite $\left(R^{i}=0\right)$, a resource ray (solid arrow), and isosurfaces $\left(f_{i}\right)$, including a zero isosurface. (c) Three resource spaces for a three-trophic level ecosystem model. Three resource rays, spanning each space from $R^{i}=1$ to $R^{i}=0$, and a planar zero isosurface $\left(f_{i}=0\right)$ are shown for each resource space. $E^{2}$ is analogous to (b) above.

Figure 2. The ecospace, resource space, resource rays, zero isosurfaces and ray gradient and ray sign conditions for logistic Lotka-Volterra (LLV) systems. (a) A one trophic level model. (b) The lower trophic level of a two-trophic level system. (c) The upper trophic level of a two-trophic level system. Solid dark axes are the axes of the ecospace $(E)$, broad dashed grey axes are the axes of the appropriate resource space $\left(E^{i}\right)$. Dashed lines are the $R^{i}=0$ lid imposed by the conservation of mass criterion. The solid arrows are the resource rays and the dotted lines denote the zero isosurfaces.

Figure 3. Resource spaces, zero isosurfaces and life histories for three trophic level Lotka-Volterra systems. (a) A logistic Lotka-Volterra system (LLV) and (b) a conservative Lotka-Volterra system (CLV). The shaded surfaces are the zero isosurfaces and the lines are typical dynamics (life histories) of each system. The resource spaces, ray gradient and ray sign conditions for each species are given in the boxes on the right. The LLV system fails the negative ray sign condition for species $x_{l}$ (panel (b), top box, third line). 
Figure 4. Resource spaces, zero isosurfaces and life histories for the Hastings and Powell (1991) model as originally formulated (a) and in mass conserving form (b). In both figure parts the model has been scaled by a nominal quantity of nutrient $\left(N_{T}=11\right)$. The shaded surfaces are the zero isosurfaces and the lines are typical dynamics (life histories) of each system that form the teacup attractor. The resource spaces, ray gradient and ray sign conditions for each species are given in the boxes on the right. The original system fails the negative ray sign condition for species $x$ (panel (a), top box, third line). 
Figure 1
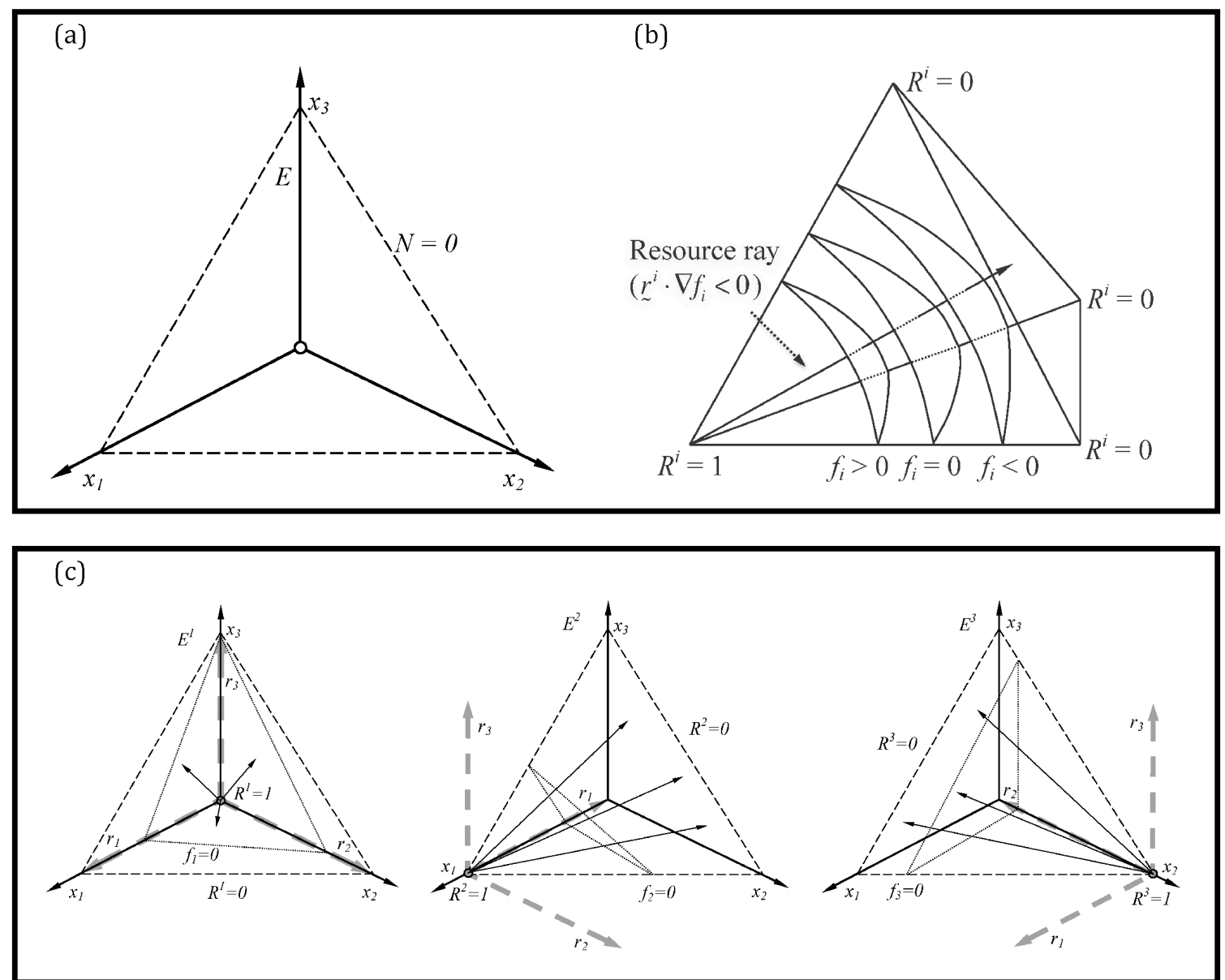
Figure 2

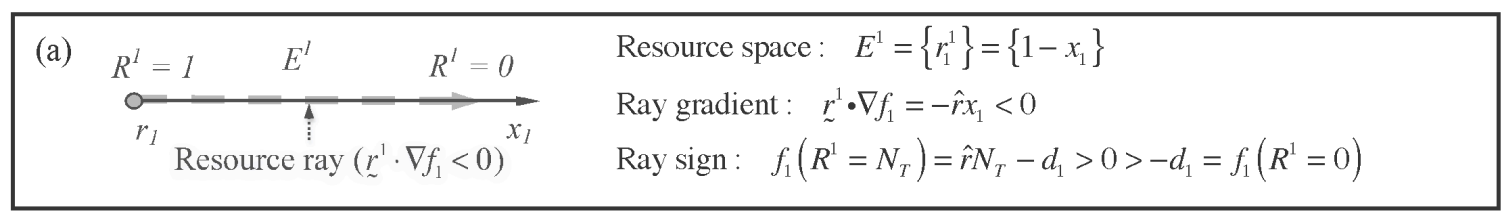
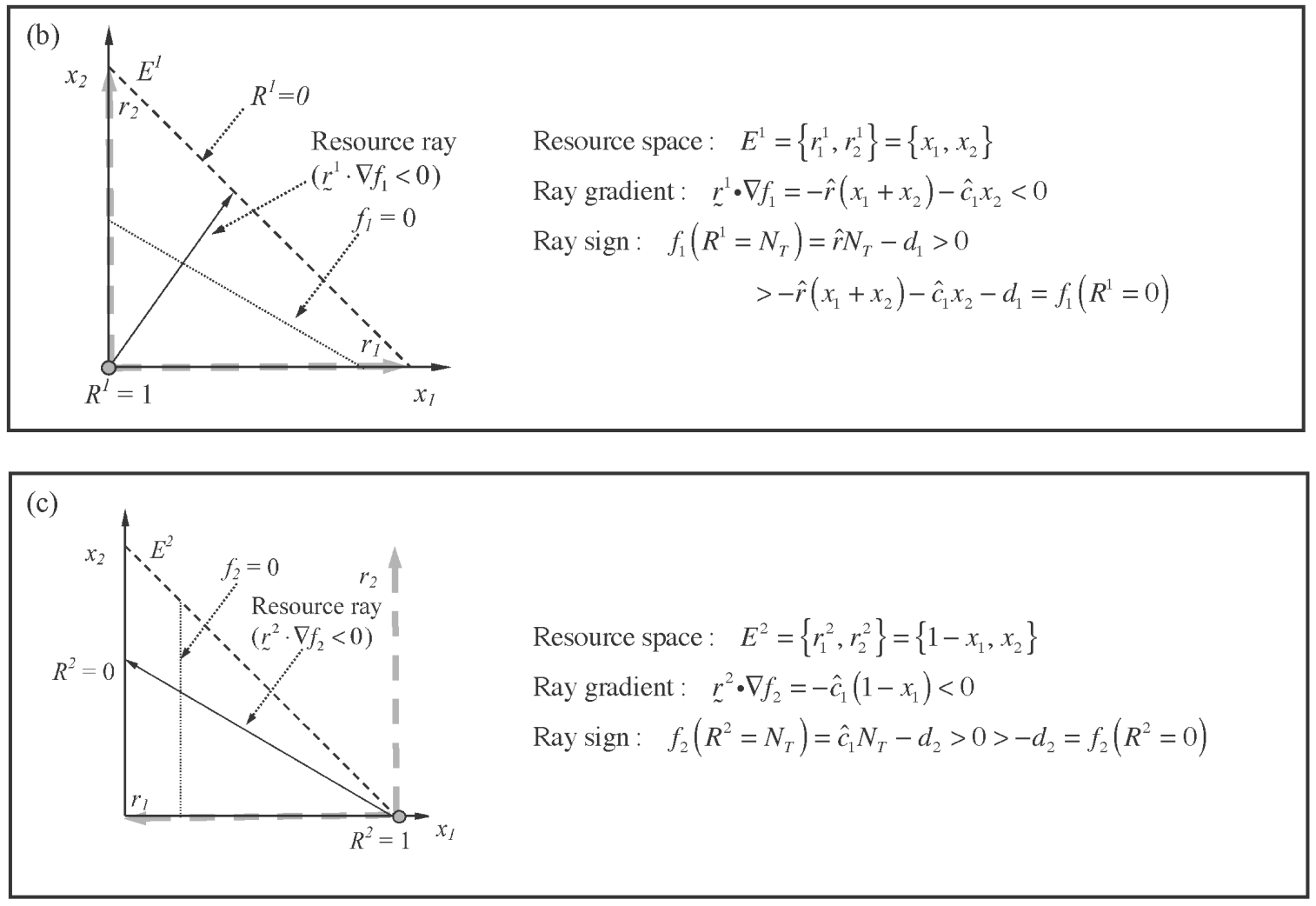


\section{Figure 3}

\begin{tabular}{|c|c|}
\hline \multirow[t]{3}{*}{ (a) } & $\begin{array}{l}\text { Resource space: } \quad E^{1}=\left\{r_{1}^{1}, r_{2}^{1}, r_{3}^{1}\right\}=\left\{x_{1}, x_{2}, x_{3}\right\} \\
\text { Ray gradient: } \stackrel{\sim}{r}^{1} \cdot \nabla f_{1}=-\hat{r}\left(x_{1}+x_{2}+x_{3}\right)-\hat{c}\left(x_{1}+x_{2}\right)<0 \\
\text { Ray sign: } f_{1}\left(R^{1}=1\right)=\hat{r}-d_{1}>0>-\hat{c}_{1} x_{2}-d_{1}=f_{1}\left(R^{1}=0\right)\end{array}$ \\
\hline & 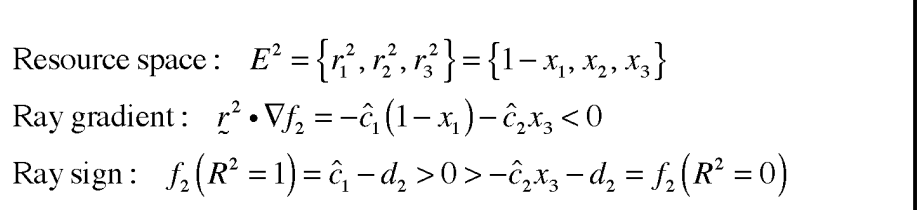 \\
\hline & 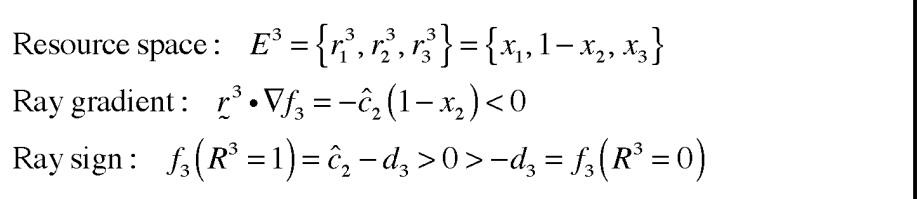 \\
\hline \multirow[t]{3}{*}{ (b) } & 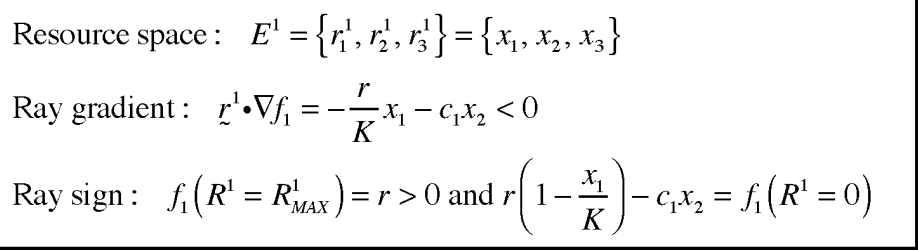 \\
\hline & 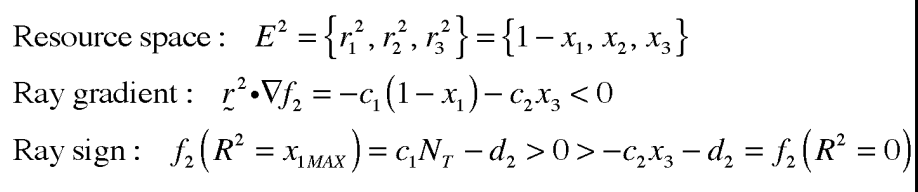 \\
\hline & 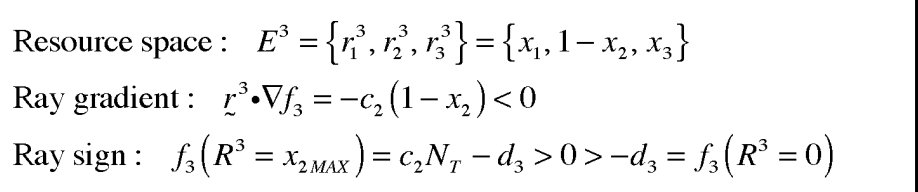 \\
\hline
\end{tabular}




\section{Figure 4}

\begin{tabular}{|l|l|l|}
\hline (a) & $\begin{array}{l}\text { Resource space: } E^{1}=\left\{r_{1}^{1}, r_{2}^{1}, r_{3}^{1}\right\}=\{x, y, z\} \\
\text { Ray gradient : } \dot{r}^{1} \cdot \nabla f_{1}=-x-\frac{a_{1} y}{\left(1+b_{1} x\right)^{2}}<0\end{array}$ \\
Ray sign: $f_{1}\left(R^{1}=R_{M A X}^{1}\right)=1>0$ and $1-x-\frac{a_{1} y}{1+b_{1} x}=f_{1}\left(R^{1}=0\right)$
\end{tabular}

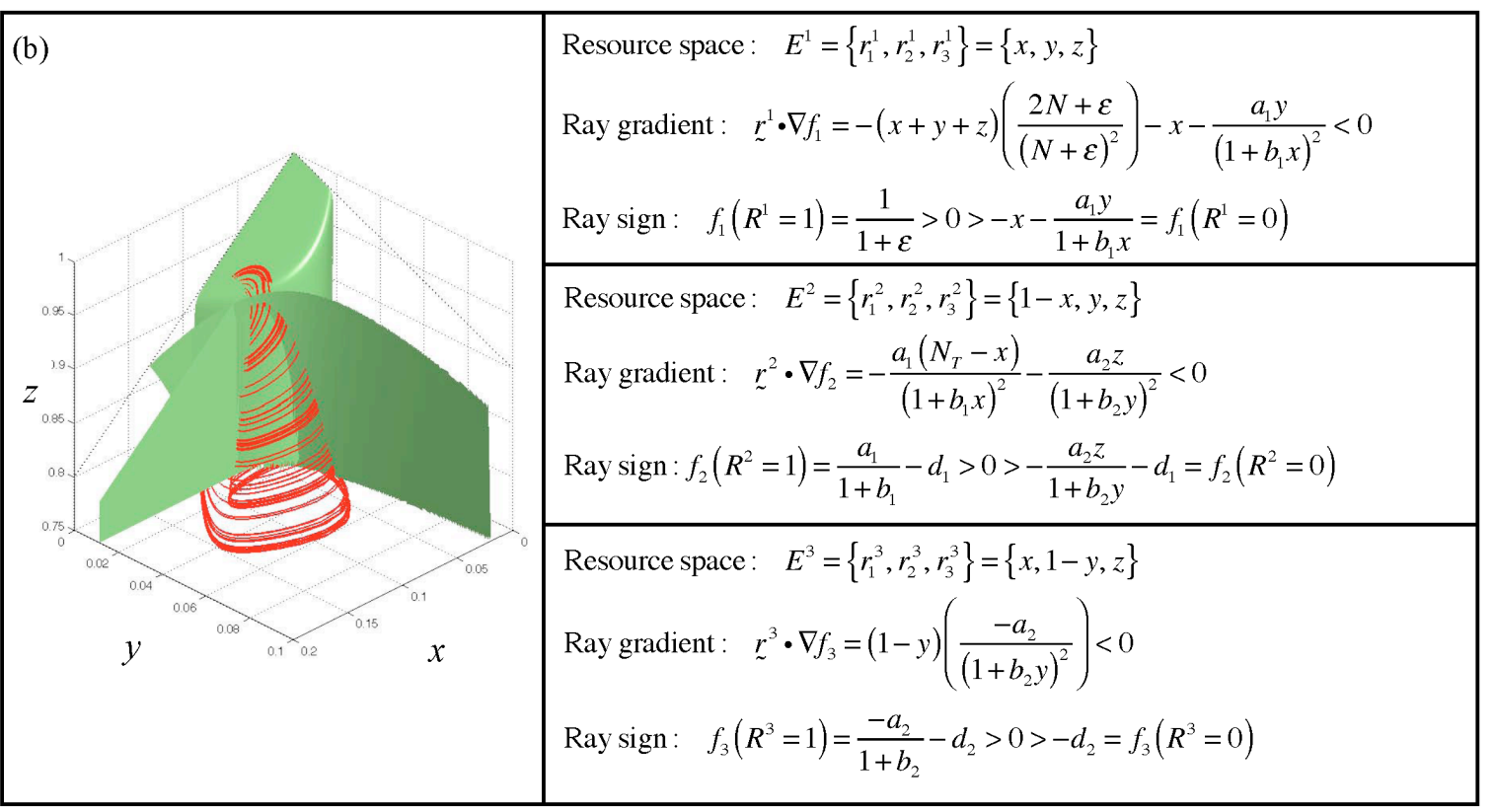

